\title{
Analysis of Real Ocean Surface Photo Imaging by Using Weighted Least Square Method
}

\author{
Muhammad Sameer Sheikh, Qunsheng Cao, and Caiyun Wang,
}

\begin{abstract}
This paper is focused on an analysis of the real images of ocean surface, such as dark ocean surface, sunlight effect on ocean surface and reflected by boat, by using twodimensional weighted least square method (2D-WLSM). We introduced three different factor values to achieve and get the maximum information of the ocean surface which contained some noisy signal. We determine the attributes of the real image using an image tool, then we convert the axes coordinates of the real image in to the pixel coordinates and obtain the pixel information of the real images by the 2DWLSM, and finally we compare the response factors of the measured values for the different cases. It is shown that the measured pixel response values are very close to the factor values and the errors between them are very small. Compared the error of the traditional LSM with WLSM we used, our method has shown better graphic results and the error are significantly reduced.
\end{abstract}

Index Terms-Image tool, ocean surface, real image, weighted least square method (WLSM).

\section{INTRODUCTION}

The research about ocean surface has started long time [1], ocean is difficult area for researchers of computer graphics. It combined together with all scale of waves [2]. The sea surface is with its complex interplay between the waves and with reflections of the sun and sky. There are several algorithms designed for modeling the surface of the sea, but it is highly complex problem. Evaluation and attributes of real images are involved by using image tool [3]. Weighted Least Square Method and Least Square Method is used to minimize the sum of the square of the error [4], [5].

Imaging radar is one kind of equipment to be used for imagining purpose is used when the radio waves reflect the object caused some changes in radio waves [6]. The return back wave is used to create images by collecting acquired data to create the $2 \mathrm{D}$ and $3 \mathrm{D}$ image. A problem occurs in many applications is how to eliminate noise from real image surface which contains some useful information. Here we have analyzed on real images pixel information by using the WLSM techniques to reduce the noise in real image surface and achieved the useful information from the real images. There is some application of weighted least square method to estimate the parameter of the surface on the digital image [7]. Some methods were proposed to de noising the digital image [8] and B-Spline shape representation [9]. Determination of radar image of ocean wave and surface

Manuscript received September 16, 2014; revised October 30, 2014.

The authors are with College of Electronics and Information Engineering, Nanjing University of Aeronautics and Astronautics, Nanjing, China (e-mail: sameer.5@hotmail.com) current by least square curve fitting [10].This paper have used the WLSM to get maximum information of the ocean surface from the photo image contained noise, by removing the noise effects in the real image of ocean surface. The image sizes and attributes have been evaluated from information of image, then converted the axes coordinates of all real images into the pixels coordinates of image. We processed pixel information which contain some noisy signal by applying 2D-WLSM, we emphasizes on real image having noisy information of image contains large errors. The error has been significantly reduced by our method and we achieved maximum useful information of images which containing some noise from the ocean surface by introducing three adjustable factor values.

\section{MATHEMATICAL METHOD}

Objective of the present work is to simulate and achieve the maximum information from photos image of the ocean surface that contain noisy information. For a general photo image, the main information processing steps are as follows:

- Using the image tool to calculate the real image attributes and information.

- Converting the axes coordinates of real images into the pixel coordinates of the images.

- Processing the pixel information of the real images used by 2D-WLSM.

- Comparing the errors of the LSM with error of the WLSM, evaluating the performance using the polynomial curve fitting.

In order to find the control parameter of the real image $\left\{\phi_{i} i=1,2, \cdots, n\right\}$, it is determined by the measured pixel values and the known values. There are two hypothesis to be considered. For the hypothesis 1, the measured pixel values are $\left\{b_{j}, j=1,2, \cdots, m\right\}$ along the vertical direction, and known pixel values are $\left\{a_{i}, i=1,2, \cdots, n\right\}$ along the horizontal direction. In contrast to the hypothesis 1 , the measured pixel values are along the horizontal direction and known pixel values are $\left\{b_{j}, j=1,2, \cdots, m\right\}$ along the vertical direction for the hypothesis 2 .

The Least Square Method is used to evaluate the control parameter $\left\{\phi_{i}\right\}$ and $\left\{\phi_{j}\right\}$ of real image, the calculation errors are as follows,

$$
\begin{aligned}
& \varepsilon_{1}=\sum_{k=1}^{m}\left[h \phi_{i}\left(a^{k}\right)-b^{k}\right]^{2} \\
& \varepsilon_{2}=\sum_{k=1}^{m}\left[h \phi_{j}\left(b^{k}\right)-a^{k}\right]^{2}
\end{aligned}
$$


where $h \phi_{i}(a)$ and $h \phi_{j}(b)$ are the predicted pixel values for two hypothesis. to get the control parameter value we do partial differential of Eq.(1) and (2), and then we obtain [9].

$$
\begin{gathered}
\phi_{i}=\left(A^{T} A\right)^{-1} A^{T} B \\
\phi_{j}=\left(B^{T} B\right)^{-1} B^{T} A
\end{gathered}
$$

In the Eq. (3), $B$ is the output pixel values of $\left\{b_{j}\right\}$ and $A$ is input sets of $\left\{a_{i}\right\}, \ldots,\left\{a_{n}\right\}$, similarly in Eq. (4), $A$ is the output pixel value of $\left\{a_{i}\right\}$ and $B$ is the input set of $\left\{b_{j}\right\}, \ldots,\left\{b_{n}\right\}$.

For two hypothesis,

$$
\begin{gathered}
h \phi_{i}(a)=\sum_{i=0}^{n-1} \phi_{i} a_{i}=\phi^{T} a \\
h_{j}(b)=\sum_{i=0}^{n-1} \phi_{j} b_{j}=\phi^{T} b
\end{gathered}
$$

where the ranges of the input parameter ranges $a$ and $b 0$ to $n-1$.

Assuminge is the minimized parameter, which is the square of the error between the predicted pixel values $h \phi_{i}(a)$ or $h \phi_{j}(b)$ and all the output $b_{j}$ or $a_{i}$ for all $K$ value for the hypothesis.

$$
\begin{aligned}
\mathcal{E} & =\frac{1}{2} \sum_{k=1}^{m} w^{k}\left[h \phi_{i}\left(a^{k}\right)-b^{k}\right]^{2} \\
& =\frac{1}{2}(A \phi-B)^{T} W(A \phi-B)
\end{aligned}
$$

Table I is listed the matrix size of input $A(i, j)$ and predicted $B(j)$ for hypothesis 1 , and input $B(i, j)$ and predicted $A(i)$ for hypothesis 2 , in which the index $n$ is the number of pixel elements and dimension is shown below.

TABLE I: THEINPUT AND OUTPUT SIZE OF DIMENSION

\begin{tabular}{ccc}
\hline \hline Hypothesis & Input & Output \\
\hline 1 & $A(i, j)[n \times n]$ & $B(j)[n \times 1]$ \\
2 & $B(i, j)[n \times n]$ & $A(i)[n \times 1]$ \\
\hline \hline
\end{tabular}

Similarly to the LSM, the minimized squared error of the real image ocean surface representation in the WLSM are as given as:

$$
\begin{aligned}
& \varepsilon_{1}=\sum_{k=1}^{m} w^{k}\left[h \phi_{i}\left(a^{k}\right)-b^{k}\right]^{2} \\
& \varepsilon_{2}=\sum_{k=1}^{m} w^{k}\left[h \phi_{j}\left(b^{k}\right)-a^{k}\right]^{2}
\end{aligned}
$$

where the parameter $w$ is the weight.

Based on the Eq. (8) and (9), the total error yields.

$$
\varepsilon=\sum_{k=1}^{m} w^{k}\left[h \phi_{i}\left(a^{k}\right)-b^{k}\right]^{2}+\sum_{k=1}^{m} w^{k}\left[h \phi_{j}\left(b^{k}\right)-a^{k}\right]^{2}
$$

In order to obtain better response of the system, the weights are chosen as follow,

$$
\begin{gathered}
w^{k}=e^{-\frac{\left(a^{k}-a\right)^{2}}{2 \tau^{2}}} \\
w^{k}=e^{-\frac{\left(b^{k}-b\right)^{2}}{2 \tau^{2}}}
\end{gathered}
$$

where the parameter $a$ and $b$ are the input pixel values, $k$ is the number of the pixels element, and $\tau$ is the adjustable factor. The weight $w$ is very important in the WLSM, depends on the adjustable factor value $\tau$.

Partial differentiate to $\varepsilon$ with respect to $\phi_{i}$ minimize the weighted least square error, so

$$
\frac{\partial \varepsilon}{\partial \phi_{i}}=\left(A^{T} W A \phi-A^{T} W B\right)
$$

It leads finally,

$$
\begin{gathered}
\phi_{i}=\left(A^{T} W A\right)^{-1} A^{T} W B \\
\phi_{j}=\left(B^{T} W B\right)^{-1} B^{T} W A
\end{gathered}
$$

\section{Process Block Diagram}

The process diagram contains of several block and each block has its own process and functionality, consider the real image of oceans surfaces such as dark ocean surface, sunlight effect on a surface and reflected by boat, captured by radar, the real image we get is noisy image in terms of dark ocean, sunlight and reflected by boat, after getting the noisy real image we find the attributes of the real image ocean's surface, secondly we convert the axes coordinate to the pixel coordinates of the image, though we get pixel information of the real image which contain some noise, and finally we apply weighted least square method (2D) by defining three different factor values (see Fig. 1).

\section{Simulation RESUlts}

First, we consider an ocean surface image, the all image information is obtained by the image tool, and the attributes are shown in Table II, which the size of height and the width of the image are 360 and 480 pixels, respectively, Fig. 2 is the original dark ocean surface in the real image.

Similarly, we can obtain successively the image information of Sun light effect on ocean and reflected image by boat on ocean. The sizes of images both are $177 \times 284$ pixels.

For any real image, we get the pixel information of all real images by converting from the axes coordinates of the real images and then processed this pixel information using by the 2D-WLSM fit to exponential by defining three factor values to achieve the more information of the real image which contain some noisy information. We compare the response between the measured pixel information and adjust factor $\tau$ values (see Fig. 1). 


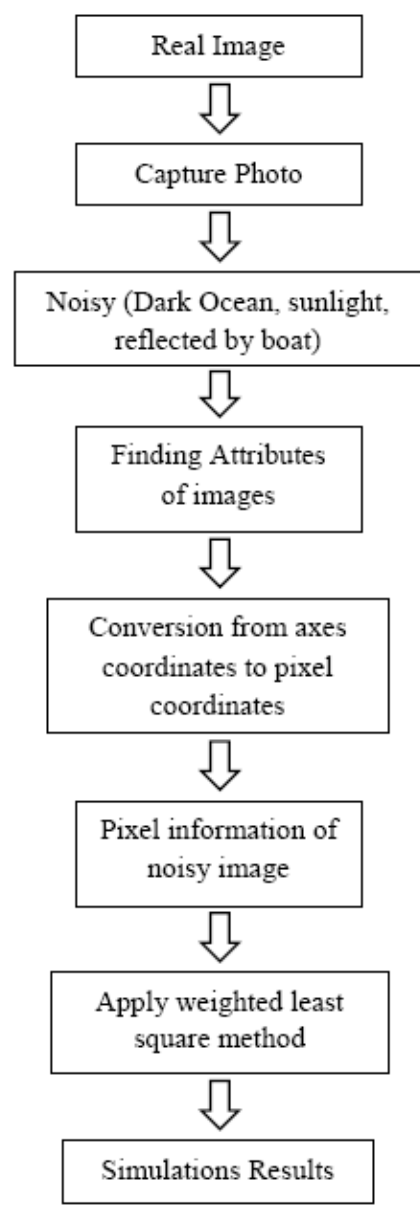

Fig. 1 Block diagram for the novel model

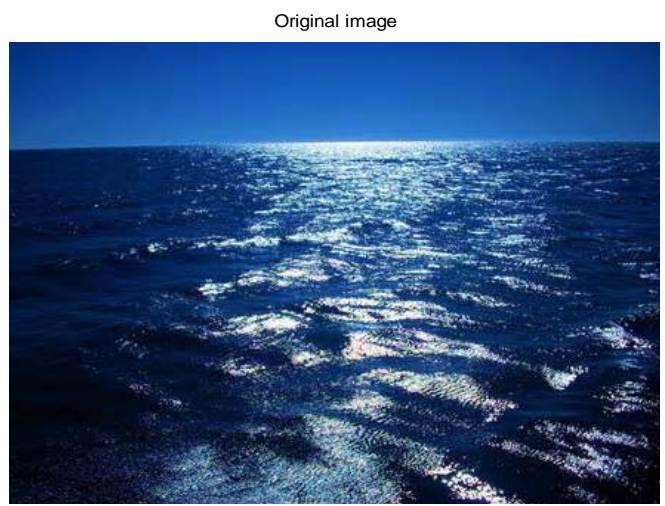

Fig. 2 Dark ocean surface real image [Internet sources].

TABLE II: IMAGE INFORMATION (IMAGE TOOL)

\begin{tabular}{cc}
\hline \hline Attributes & Value \\
\hline Width (columns) & 480 \\
Height (rows) & 360 \\
Class & Unit 8 \\
Image type & true color \\
\hline \hline
\end{tabular}

Eq. (11) and (12) are used to determine the weight of the system, Eq. (14) and (15) are used to find value of $\Phi_{i}$ and $\Phi_{j}$ which are used for the both hypothesis to minimize the error between the measured pixel information and $\tau$ values.

Fig. 3 are the comparisons of the response of the dark ocean surface real image for different factor $\tau$ values and measured pixel values by simulating. It has shown that the two responses are nearly same, which means that our measured pixel values are very close to all factor values. From Fig. 3(a), when factor $\tau$ increased, the measured pixel response is nearly the same of factor values.

In Fig. 3(b) there is slightly difference between two responses. Because the measured pixel value and factor values were primarily caused by the weighting function and the input parameter when it approaching to $\tau=25$ the measured pixel response has some resemblance of factor response, and the calculatederror is less when applied the WLSM by defining three different factor values.

Now consider the sunlight effect on the ocean surface in real image shown in Fig. 4, the size of image has been calculated using by the image tool.

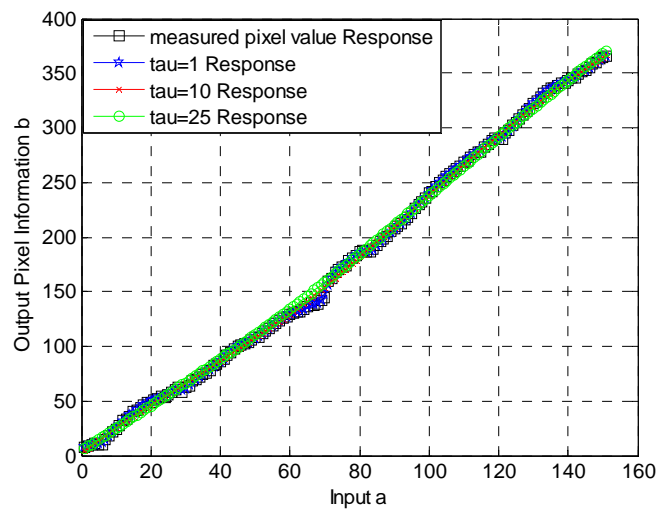

(a)

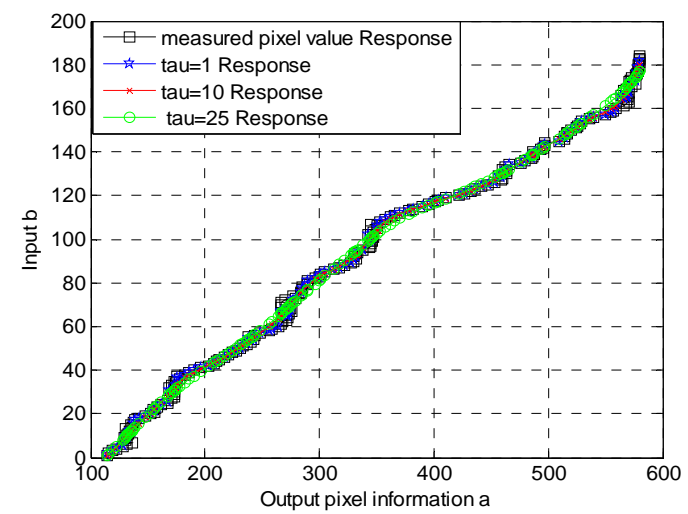

(b)

Fig. 3. Dark ocean surface response,(a)for hypothesis 1, (b) for hypothesis 2.

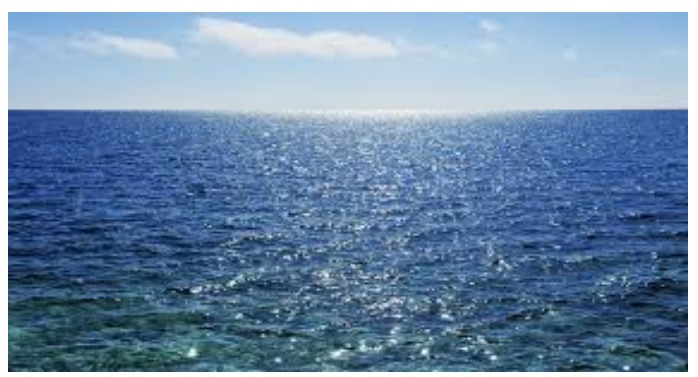

Fig. 4. Sun light on the ocean surface real image [internet sources]

Fig. 5 are the comparisons of the response of the ocean surface with sunlight to the real image corresponding different factortvalues and measured pixel values by simulating.

Fig. 5(a) is presented that in the early time region there has slightly difference between the measured pixel value and the factor values because of the sun light effect and the factor value, when the $t$ factor is increased to 10 the measured 
pixel response is quite closer to the factor response and smoothly, while in Fig. 5(b) is slightly differ at initially when the response started because of initial weights and factor value but when it is approaching toward $\tau=25$ the measured pixel response of sunlight image is close to the factor value response and the error between them is less.

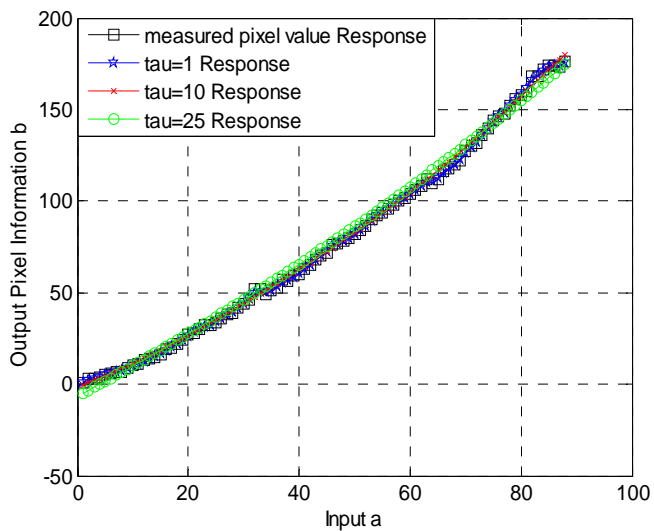

(a)

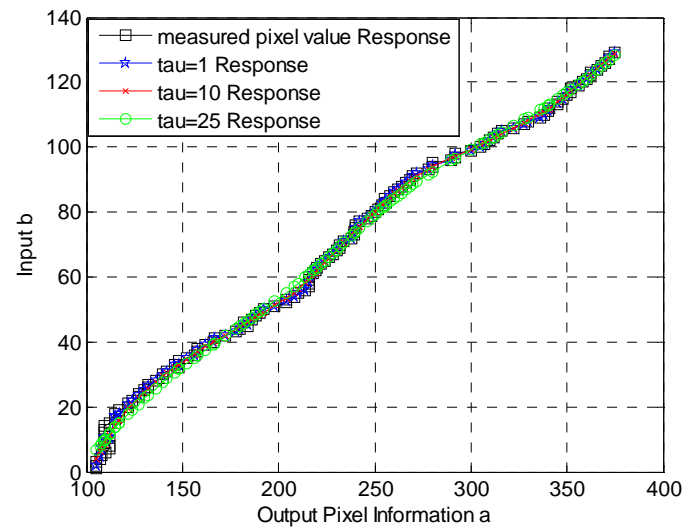

(b)

Fig. 5. Ocean surface response with sunlight effect, (a) for hypothesis 1, (b) for hypothesis 2 .

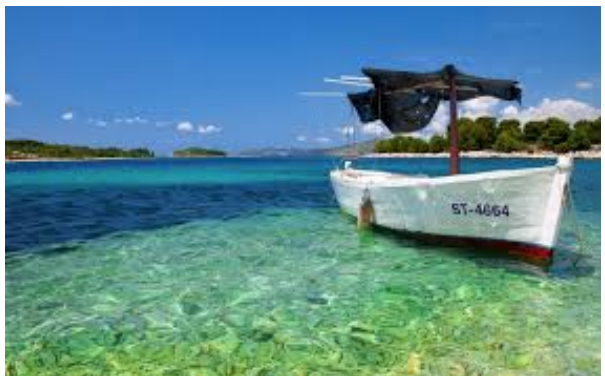

Fig. 6. Reflected image by a boat [internet sources].

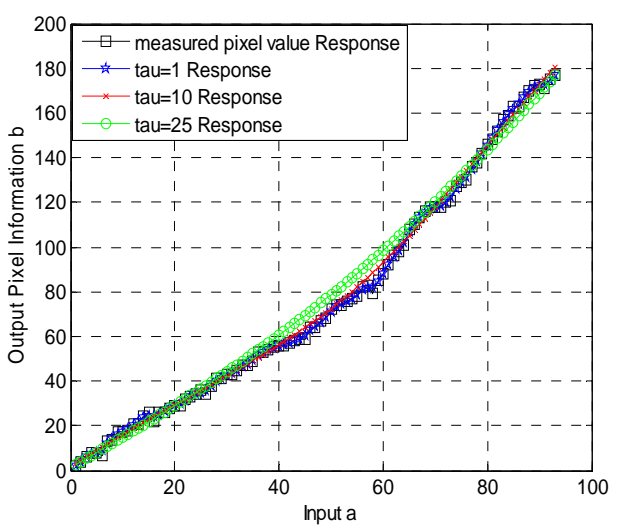

(a)

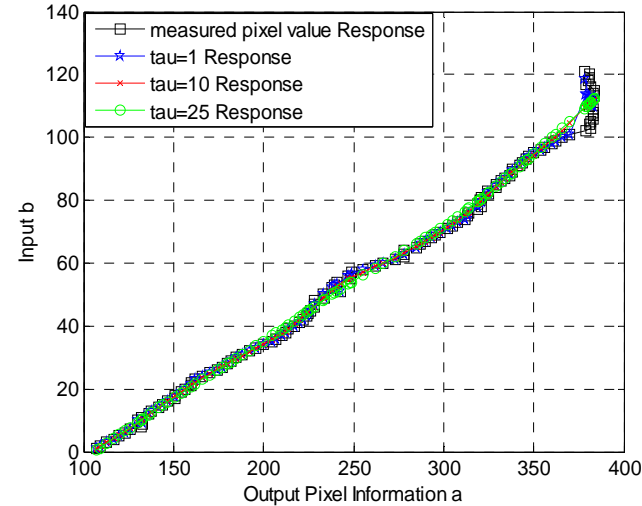

(b)

Fig. 7. Ocean surface response withboat, (a) for hypothesis 1, (b) for hypothesis 2 .

Finally, we consider the reflected image by a boat on the ocean surface shown in Fig. 6.

Fig. 7 is the comparisons of the response of the reflected image by a boat for different factor $\tau$ values and the measured pixel value by simulating.

Fig. 7(a) is shown the measured pixel values at the starting stage are nearly identical to the factor value, at the middle stage the measured pixel values response are slightly differ from factor response because of the reflected object and the increased weights. When the factor value $\tau$ chosen as 25 , the measured pixel values response of the boat are nearly same the factor response and the error between them is less.

Fig. 7(b) shows that the measured pixel value and the factor value are identical when the weight is low. At the middle stagethe measured responses are slightly differ from the factor value because of reflected object and increasing weight. When $\tau=25$, the measured response is closely identical to the factor response and the calculated error between them is minimized.

For two hypothesis in order to compare the errors between the LSM and the WLSM, consider 10 pixel values $(k=10)$ for each image such as dark image, sun light image and reflected image. For hypothesis 1 and hypothesis 2, the errors are given in Table III and Table IV, respectively, the weighted LSM has less error than the LSM.

TABLE III: APPROXIMATION ERROR OF HYPOTHESIS 1

\begin{tabular}{ccc}
\hline \hline Images & LSM & WLSM \\
\hline Dark Image & 6.8 & 0.087 \\
Sun Light Image & 49.38 & 9.43 \\
Reflected Boat & 30.3 & 10.88 \\
\hline \hline
\end{tabular}

TABLE IV: APPROXIMATION ERROR OF HYPOTHESIS 2

\begin{tabular}{ccc}
\hline \hline Images & LSM & WLSM \\
\hline Dark Image & 7.2 & 0.763 \\
Sun Light Image & 27.2 & 2.59 \\
Reflected Boat & 49.95 & 15.9 \\
\hline \hline
\end{tabular}

Fig. 8 shows that evaluation of the performance of the method, the curve of the simulation result by the WLSM and the curve of the second-order polynomial are fitting. In Fig. 8 at the beginning stage there is little difference between the measured value and the curve response, but at the middle 
and final stage there is some resemblance between two curves. It is noted that our measured values are close to the curve response and the errors between them are less.

Fig. 9 displays the error comparison between the LSM and the WLSM techniques by considering the image reflected by boat for hypothesis 1 evaluate the error values response betweenthe LSM and the WLSM. It has foundthat the error is reduces significantly in the WLSM, at the same time, the spikes in the error curve have been reduced obviously byusing our proposed method.

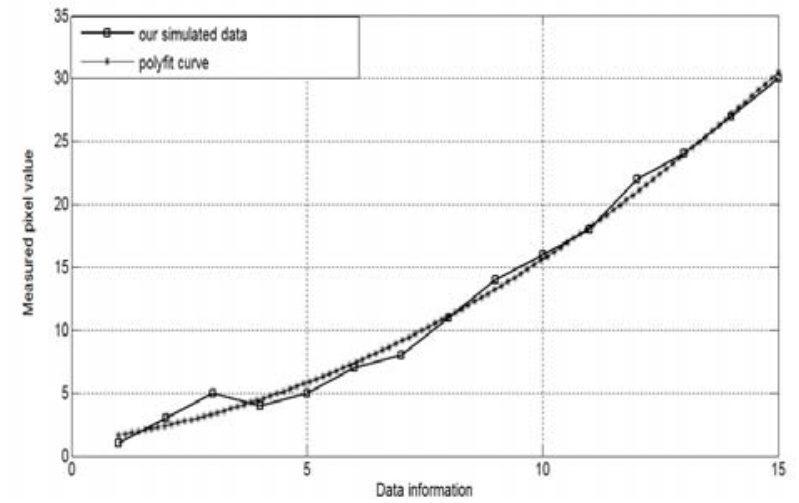

Fig. 8. Comparison of the simulation result andsecond-order polynomial

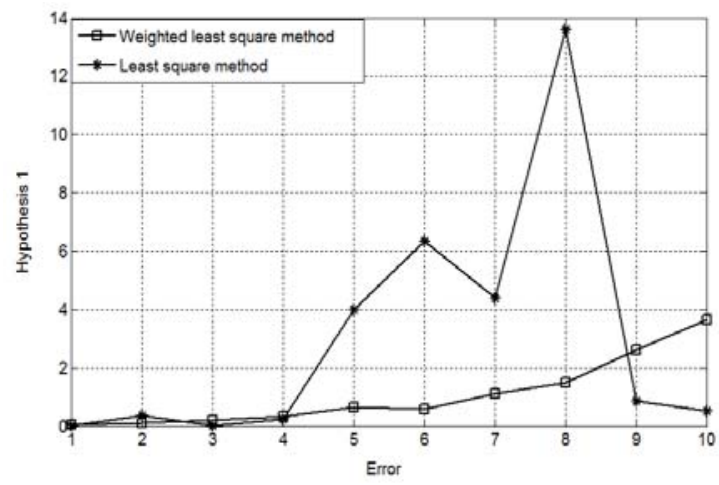

Fig. 9. Error comparison between LSM and WLSM.

\section{CONCLUSIONS}

In this paper, the ocean surface real images have been studied firstly based on the WLSM for two assumed hypothesis. Three adjustable factor values $\tau$ have been introduced for both hypothesis and the responses of the real image ocean surface have been evaluated. It has found that the measured pixels values response are very close to the $\tau$ value response, we have achieved the maximum information of the ocean surface which contain noisy information's and the ocean surface errors have been minimized. The error comparison between the LSM and the WLSM has been done and as well the comparison of our simulated data with polynomial curve fitting.

\section{ACKNOWLEDGMENT}

This work is supported by Key Laboratory of Radar Imaging and Microwave Photonics Ministry of Education, Nanjing University of Aeronautics and Astronautics, Nanjing, 210016 People Republic China.

\section{REFERENCES}

[1] N. Y. Yeo and S. H. Park, "Analysis and realization of ocean wave surface by utilizing matlab," Korea Institute of Electronics and Telecommunications, vol. 5, no. 3, June 2010

[2] E. Bruneton and F. Neyret, and N. Holzschuch, "Real time realistic ocean lighting using seamless transition from geometry to BRDF," Implemented in the Open-Source Terrain Library Proland, The Eurographic Association and Blackwell Publishing, vol. 29, no. 2, pp. 487-496, 2010

[3] R. C. Gonzalez, E. Richard, and L. Steven, Digital Image Processing Using MATLAB, Gatesmark Publishing, 2009

[4] Weighted Least Square Estimation techniques and multiple $\begin{array}{lll}\text { regressions } & \text { Stat. } & \text { [Online]. }\end{array}$ http://www.stat.nus.edu.sg/ staxyc/REG33. PDF

[5] D. C Agnew and C Constable, Least Square Estimation, 2008, pp. 713

[6] Igpphome.

[Online].

Available: http://igpphome.ucsd.edu/ cathy/Classes/SIO223A/sio223a.chap7.pdf

[7] Wikipedia the Encyclopedia. [Online]. Available: http://www.en.wikipedia.org/wiki/Main_Page

[8] Radar imaging - Wikipedia, the free encyclopedia. [Online]. Available: http://www. en.wikipedia.org/wiki/Radar_imaging.

[9] Y. Cheng, T. Liang, and L. Shing, "A weighted least square approach for B-spline Shape Representation," Communications, Control and Signal Processing, pp. 894-898, 2008.

[10] M. Tico and P. Kuosmanen "Weighted least squares for the approximation of directional derivatives," Acoustics speech and signal processing, vol. 3, pp. 1681-1684, 2001.

[11] K. Hirakawa and T. Parks, "Image denoising using total least square," IEEE Transaction on Image Processing, vol. 15, pp. 2730-2742, 2006.

[12] I. R Young, W. Rosenthal, and F. Ziemer, "A three dimensional analysis of marine radar images for the determination of ocean wave directionally and surface currents," Journal of Geophysical Research, vol. 90, pp. 1049-1059, 1985.

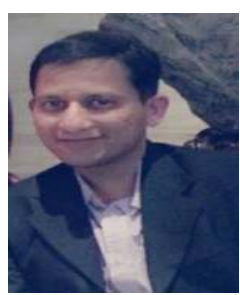

Muhammad Sameer Sheikh was born in Karachi Pakistan, he received his bachelor degree in electronic engineering from Hamdard University, Karachi, Pakistan in 2007. He received his master degree in telecommunication engineering from Hamdard University Karachi, Pakistan in 2010. He is currently a $\mathrm{PhD}$ candidate at College of Electronics and Information Engineering, Nanjing University of Aeronautics and Astronautics. Nanjing, China. his main research interest focused on the area of computational electromagnetics, radar signal processing, image processing and digital signal processing.

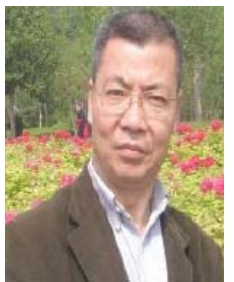

Qunsheng Cao received his Ph.D. in electrical engineering from Hong Kong Polytechnic University in 2001. From 2001 to 2005 he worked as a research associate in the Department of Electrical Engineering, University of Illinois at Urbana-Champaign and at the Army High Performance Computing Research Center (AHPCRC), University of Minnesota. In 2006, Dr. Cao joined the Nanjing University of Aeronautics and Astronautics (NUAA), China, as a Professor of electrical engineering. Dr. Cao's current research interests are in computational electromagnetics, antenna and microwave technology and the radar signal processing. Dr. Cao has published more than 120 academic papers in refereed journals and conference proceedings.

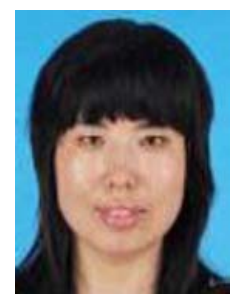

Caiyun Wang was born in Shanxi, China, on September 30, 1975. She graduated in 1996 with a B.S. degree and in 1999 with a M.S. degree. She received the $\mathrm{Ph} . \mathrm{D}$. degree in signal and information processing from Beihang University, Beijing, China, in 2008. She is currently an associate professor with the College of Astronautics, Nanjing University of Aeronautics and Astronautics (NUAA). Her major research interests are in the fields of radar automatic target recognition (RATA), radar signal processing, image processing, digital signal processing and compress sensing. 